\title{
The Equilibrium Between Elementary Sulfur and Aqueous Polysulfide Solutions
}

\begin{abstract}
A N T S TEDER
Swedish Forest Products Research Laboratory (STFI), P.O.B. 5604, S.114 86 Stockholm, Sweden

The equilibrium between elementary sulfur and aqueous sodium polysulfide has been studied at 25 and $80^{\circ} \mathrm{C}$ using two methods, firstly, by adding acid to polysulfide solutions until precipitation of elementary sulfur, and secondly, by dissolving elementary sulfur in aqueous polysulfide solution until equilibrium is established. The stoichiometric composition of a polysulfide solution, i.e. the ratio between $S(0)$ and $S(-\mathbf{I I})$, is strongly dependent on the alkalinity. Equilibrium analysis of the results obtained has shown that the mean size of the polysulfide ions $\mathrm{S}_{n} \mathrm{~S}^{2-}$ in equilibrium with elemental sulfur is independent of alkalinity, but that the concentration of HS $^{-}$ions increases inversely with the alkalinity. From the results obtained, in combination with those of a previous spectrophotometric study, the constants for the equilibrium between elemental sulfur and polysulfide ions of varying size have been calculated.
\end{abstract}

The equilibrium of the system $\mathrm{Na}_{2} \mathrm{~S}-\mathrm{S}-\mathrm{H}_{2} \mathrm{O}$ has previously been studied 1 at different temperatures. ${ }^{1}$ The formation of thiosulfate by polysulfide degradation and the effect of alkalinity on the equilibrium have, however, not been taken into account. Since the equilibria between polysulfide ions of different size in aqueous solutions strongly depend on the alkalinity of the solution, ${ }^{2}$ according to

$$
\mathrm{S}_{m+n} \mathrm{~S}^{2-}+\mathrm{HS}^{-}+\mathrm{OH}^{-} \rightleftharpoons \mathrm{S}_{n} \mathrm{~S}^{2-}+\mathrm{S}_{m} \mathrm{~S}^{2-}
$$

an effect of alkalinity on the stoichiometric composition of an aqueous polysulfide solution in equilibrium with elementary sulfur should be expected.

The aim of the present investigation was to study the alkalinity dependence of the equilibrium between elementary sulfur and aqueous polysulfide solutions at 25 and $80^{\circ} \mathrm{C}$. The point of equilibrium between aqueous polysulfide solutions and elementary sulfur was determined by decreasing the alkalinity of the solutions at different $X_{\mathrm{s}}$ (ratio $\mathrm{S}(0) / \mathrm{S}(-\mathrm{II})$ ) until elementary sulfur was precipitated, as well as by dissolving elementary sulfur in aqueous polysulfide solutions, of controlled and comparatively low alkalinity, until equilibrium was established. The equilibria between elementary sulfur, hydrogen sulfide 
ions, and various polysulfide ions will be discussed in the light of the results obtained both in the present work and in a previous spectrophotometric study ${ }^{2}$ of equilibrated aqueous polysulfide not containing elementary sulfur.

\section{SYMBOLS}

$$
\begin{aligned}
& \text { Sulfide sulfur }=\mathrm{S}(-\mathrm{II}) \\
& \text { Polysulfide excess sulfur }=\mathrm{S}(0) \\
& {[\mathrm{S}(-\mathrm{II})]=\left[\mathrm{HS}^{-}\right]+\left[\mathrm{S}^{2-}\right]+\sum\left[\mathrm{S}_{n} \mathrm{~S}^{2-}\right]} \\
& {[\mathrm{S}(0)]=\sum n\left[\mathrm{~S}_{n} \mathrm{~S}^{2-}\right]} \\
& X_{\mathrm{s}}=\frac{[\mathrm{S}(0)]}{[\mathrm{S}(-\mathrm{II})]} \\
& \bar{n}=\frac{\sum n\left[\mathrm{~S}_{n} \mathrm{~S}^{2-}\right]}{\sum\left[\mathrm{S}_{n}^{-} \mathrm{S}^{2-}\right]} \\
& \bar{n}_{\mathrm{e}}=\bar{n} \text { at equilibrium with elemental sulfur } \\
& B=\left[\mathrm{HS}^{-}\right]\left[\mathrm{OH}^{-}\right] /[\mathrm{S}(0)] \\
& B_{\mathrm{e}}=B \text { at equilibrium with elemental sulfur }
\end{aligned}
$$

\section{EXPERIMENTAL}

Chemicals. The solutions were prepared as previously described. ${ }^{3,4}$ Granulated sulfur from the Swedish Shale Oil Co., Kvarntorp, was used. This was found to contain less interfering impurities than did reagent grade sulfur available from other sources. All the solutions studied were $3 \mathrm{M}$ in Na+ with $\mathrm{Cl}^{-}$as the inert anion. This high ionic strength was chosen in order to be able to express the equilibria in terms of concentration. This high ionic strength was also used in previous spectrophotometric studies on polysulfide equilibria ${ }^{2}$ and in general studies on the chemistry of polysulfide pulping. ${ }^{5}$

Analyses. The $\mathrm{OH}^{-}$concentration of the solutions studied was determined at the two temperatures with glass electrodes calibrated against solutions of known $\mathrm{OH}^{-}$concentration and the same ionic strength. ${ }^{4}$ Sulfide sulfur, S(-II), and polysulfide excess sulfur, $\mathrm{S}(0)$, were determined by an acidimetric method. ${ }^{6}$ For control purposes, the excess sulfur content was also determined spectrophotometrically. ${ }^{\text {? }}$

Apparatus. The experiments were carried out in a closed thermostated double-walled Pyrex vessel under purified nitrogen. The solution was stirred by a Teflon encapsulated magnetic stirrer. In experiments with aqueous polysulfide solutions it is essential to avoid all contact with air, as polysulfide ions rapidly react with oxygen. The lid of the vessel was equipped with standard ground-glass joints, which were used for immersing in the polysulfide solutions: a glass electrode, an $\mathrm{Ag}-\mathrm{AgCl}$ reference electrode, a thermometer, and a capillary connected to a piston burette used for the addition of solutions.

\section{Equilibrium studies}

(a) By additions of acid. The point of equilibrium between elemental sulfur and polysulfide solutions of different $X_{s}$ was determined by decreasing the alkalinity of polysulfide solutions, not in equilibrium with elemental sulfur, by successive additions of $\mathrm{HCl}$, until elemental sulfur was formed. After each addition, the $\mathrm{OH}^{-}$concentration of the solution was determined. The alkalinity obtained was plotted against the volume of acid added. The volume at which equilibrium with elemental sulfur was established

Acta Chem. Scand. 25 (1971) No. 5 
was observed as an inflection in the titration curve (Fig. 1). A permanent opalescence appeared at approximately the same alkalinity. The polysulfide solutions can be considered as being in equilibrium during the titration, since the rearrangement reactions between polysulfide ions of different sizes, hydrogen sulfide, and hydroxide ions are fast. $^{8}$ In most experiments, an $[\mathrm{S}(0)]$ of $0.1 \mathrm{M}$ was used. With increased $X_{\mathrm{s}}$ the point of equilibrium with elemental sulfur became more difficult to detect. This method thus was unsuitable for polysulfide solutions with $X_{\mathrm{s}}$ above 4 .

(b) By additions of sulfur. The point of equilibrium between elemental sulfur and polysulfide solutions of various alkalinities was determined by dissolving elemental sulfur in the solutions at constant alkalinity until equilibrium $X_{\mathrm{s}}$ was reached. Granulated elemental sulfur was added in excess to a polysulfide solution of $X_{s} \sim 4$. The temperature was then raised to the desired level. The alkalinity was held at a constant, predetermined value with an automatic titrator throughout the reaction. ${ }^{9}$ Liquor aliquots were removed after various time intervals and thus separated from the elemental sulfur. Immediately after the withdrawal, the samples were diluted with an $\mathrm{HS}^{-}$solution of known concentration, thus eliminating the risk for precipitation of elemental sulfur during the cooling and subsequent analysis for sulfide and polysulfide excess sulfur. From the analysis of the diluted samples the $X_{\mathrm{s}}$ in the original sample could be calculated. At the point where the analyses indicated that the $X_{3}$ did not increase further, equilibrium was considered to be reached. The reaction time required to reach equilibrium was about $24 \mathrm{~h}$

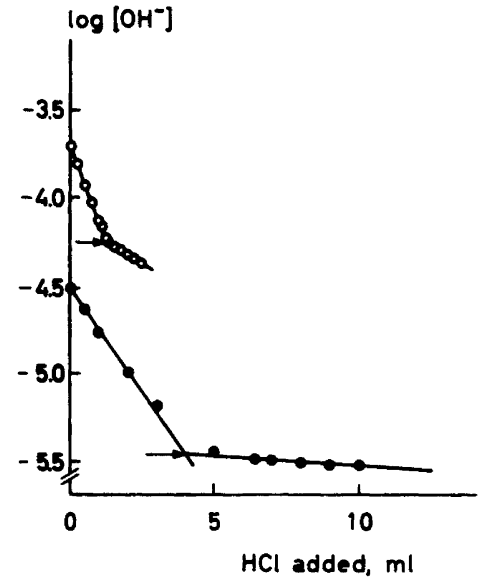

Fig. 1. An example of the method of decreased alkalinity for the determination of the point of equilibrium between an aqueous polysulfide solution and elementary sulfur. The measured alkalinity of polysulfide solutions, of $X_{\mathrm{s}}=1$ (O) and $\mathrm{X}_{\mathrm{s}}=3.5$ (O), respectively, is plotted against the volume of acid added. The points at which equilibrium with elementary sulfur was considered obtained, is indicated with arrows. Temperature $=25^{\circ} \mathrm{C}$.

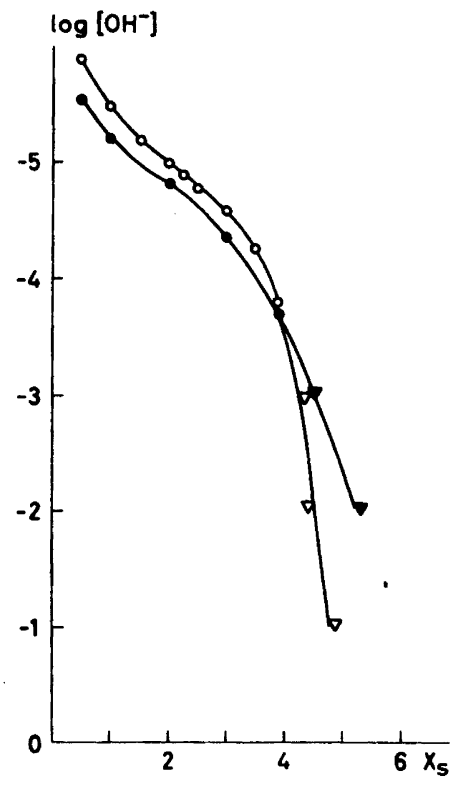

Fig. 2. The relationship between $X_{\mathrm{s}}$ and alkalinity for aqueous polysulfide solutions in equilibrium with elementary sulfur.

\begin{tabular}{|c|c|c|}
\hline \multirow{2}{*}{ Temp. } & $\begin{array}{c}\text { Decreased } \\
\text { alkalinity }\end{array}$ & $\begin{array}{c}\text { Increased } \\
X_{\mathbf{8}}\end{array}$ \\
\hline $25^{\circ} \mathrm{C}$ & 0 & $\nabla$ \\
$80^{\circ} \mathrm{C}$ & 0 & $\nabla$ \\
\hline
\end{tabular}

Acta Chem. Scand. 25 (1971) No. 5 
at $25^{\circ} \mathrm{C}$, and about $0.5 \mathrm{~h}$ at $80^{\circ} \mathrm{C}$. Part of the polysulfide underwent thermal decomposition during the reaction to hydrogen sulfide and thiosulfate ions. Polysulfide solutions of high $X_{\mathrm{s}}$ and high alkalinity are especially unstable. ${ }^{3,9}$ In order to avoid excessive ( $>20 \%$ ) thermal polysulfide degradation, the hydroxide ion concentration was limited to $\leq 0.1 \mathrm{M}$ at $25 \% \mathrm{C}$, and to $\leq 0.01 \mathrm{M}$ at $80^{\circ} \mathrm{C}$. At a given distribution of polysulfide ions of various sizes, the rate of thermal polysulfide degradation is usually directly proportional to the alkalinity. ${ }^{9}$ As this method of equilibrium study was found to be less accurate than that described above, it was only used for $X_{\mathrm{s}}$-values above 4 . In most of the experiments, the final excess sulfur concentration, $[\mathbf{S}(0)]$, was about $0.5 \mathbf{M}$.

\section{RESULTS AND DISCUSSION}

The effect of alkalinity. The ratio polysulfide excess sulfur to sulfide sulfur, $X_{\mathrm{s}}$, of aqueous polysulfide solutions in equilibrium with elementary sulfur was found to increase considerably with alkalinity (Fig. 2). It is thus not correct to neglect the effect of alkalinity, as has been done in a previous study by Arntson et al. ${ }^{1}$ At high alkalinities, the equilibrium $X_{\mathrm{s}}$, at a given alkalinity, seems to vary directly with the temperature, while at low alkalinities an inverse relationship is observed.

Polysulfide solutions with $X_{\mathrm{s}}$ and alkalinity combinations below those of the equilibrium curve in Fig. 2 are stable, apart from thermal decomposition, ${ }^{9}$ in the absence of elementary sulfur. Solutions with $X_{\mathrm{s}}$ and alkalinity combinations above those of the equilibrium curve are unstable and tend to precipitate elementary sulfur.

Changes in the polysulfide excess sulfur concentration and sulfide sulfur concentration did not seem to affect the alkalinity $-X_{\mathrm{s}}$ relationship. This result is expected, as it previously has been shown that the relative amounts of the different polysulfide ions are not effected by concentration changes, provided the $X_{\mathrm{s}}$ and the alkalinity are kept constant. ${ }^{2}$

The mean size of polysulfide ions. When the concentrations of sulfide and hydrogen polysulfide ions can be neglected, which has been shown ${ }^{2,8}$ to be the case in the range $-4<\log \left[\mathrm{OH}^{-}\right]<-1$, the relationship between $X_{\mathrm{s}}$ and $\bar{n}$, the mean number of excess sulfur atoms per polysulfide ion, can be expressed as

$$
1 / X_{\mathrm{s}}=B \cdot 1 /\left[\mathrm{OH}^{-}\right]+1 / \bar{n}
$$

where $B=\left[\mathrm{HS}^{-}\right]\left[\mathrm{OH}^{-}\right] /[\mathrm{S}(0)] . B$ has previously been found to be the variable that determines the relative amounts of the various polysulfide ions. ${ }^{2}$

The equilibrium between elementary sulfur and polysulfide ions of different size can be written as

$$
i \mathrm{~S}(\mathrm{~s})+\mathrm{HS}^{-}+\mathrm{OH}^{-} \rightleftharpoons \mathrm{S}_{i} \mathrm{~S}^{2-}+\mathrm{H}_{2} \mathrm{O}
$$

with the equilibrium constant

$$
K_{i}=\frac{\left[\mathrm{S}_{i} \mathrm{~S}^{--}\right]}{(\mathrm{S}(\mathrm{s}))^{i}\left[\mathrm{HS}^{-}\right]\left[\mathrm{OH}^{-}\right]}
$$

When elementary sulfur is present, the activity of sulfur, $(\mathrm{S}(\mathrm{s}))$, is unity and the equilibria can be expressed as

$$
\left[\mathrm{HS}^{-}\right]\left[\mathrm{OH}^{-}\right]=\frac{\left[\mathrm{S}_{1} \mathrm{~S}^{2-}\right]}{K_{1}}=\frac{\left[\mathrm{S}_{2} \mathrm{~S}^{2-}\right]}{K_{2}}=\ldots=\frac{\left[\mathrm{S}_{i} \mathrm{~S}^{2-}\right]}{K_{i}}=\ldots
$$

Acta Chem. Scand. 25 (1971) No. 5 
Consequently, and providing that hydrogen polysulfide ions are absent, $B=\left[\mathrm{HS}^{-}\right]\left[\mathrm{OH}^{-}\right] /[\mathrm{S}(0)]$ is constant for all polysulfide solutions at a given temperature and in equilibrium with elementary sulfur. The relative amounts and the mean size of the various polysulfide ions can thus be expected to be constant for all polysulfide solutions in equilibrium with elementary sulfur. When $1 / X_{\mathrm{s}}$ is plotted against $1 /\left[\mathrm{OH}^{-}\right]$for solutions in equilibrium with elementary sulfur, straight lines are obtained (Fig. 3). This is to be expected from eqn. (2). The intercept gives the value of $1 / \bar{n}_{\mathrm{e}}$, and the slope the value for $B_{\mathrm{e}}\left(\bar{n}_{\mathrm{e}}\right.$ and $B_{\mathrm{e}}$ are the values of $\bar{n}$ and $B$ at equilibrium with elementary sulfur).

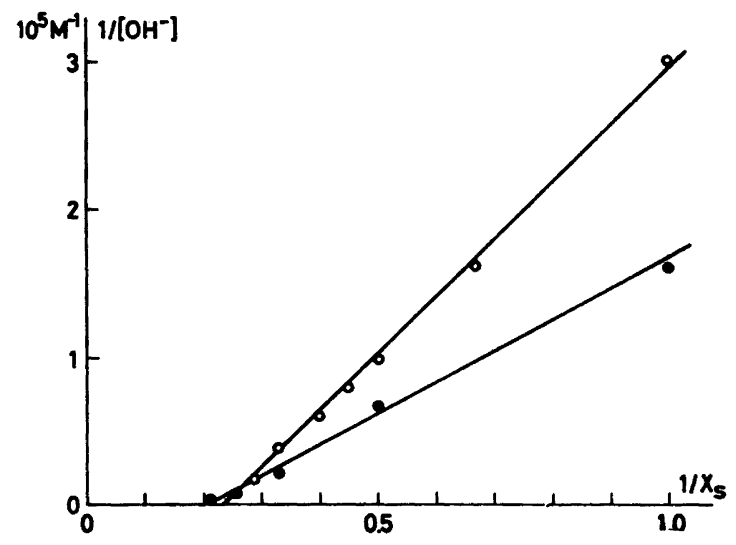

Fig. 3. Plot used for the determination of $\bar{n}_{\mathrm{e}}$ and $B_{\mathrm{e}}$, i.e. the mean size, $\bar{n}$, of the polysulfide ions, $\mathbf{S}_{n} \mathrm{~S}^{2-}$, and the parameter $B=\left[\mathrm{HS}^{-}\right]\left[\mathrm{OH}^{-}\right] /[\mathrm{S}(0)]$, for aqueous polysulfide solutions of $25^{\circ} \mathrm{C}(0)$ and $80^{\circ} \mathrm{C}(O)$ in equilibrium with elementary sulfur. According to eqn. (2), the intercept gives the value of $1 / \bar{n}_{\mathrm{e}}$, and the slope the value for $B_{\mathrm{e}}$.

According to this method, $\bar{n}_{\mathrm{e}}$ is 4.4 at $25^{\circ} \mathrm{C}$, and 5.0 at $80^{\circ} \mathrm{C}$, and $B_{\mathrm{e}} 2.5 \times 10^{-6} \mathrm{M}$ at $25^{\circ} \mathrm{C}$, and $5 \times 10^{-6} \mathrm{M}$ at $80^{\circ} \mathrm{C}$ for polysulfide solutions in equilibrium with elementary sulfur. The increase in both $\bar{n}_{\mathrm{e}}$ and $B_{\mathrm{e}}$ with temperature explains the opposite effect of temperature on $X_{\mathrm{s}}$ of solutions in equilibrium with elementary sulfur at low and high alkalinities. The increase in the mean size of the polysulfide ions in corresponding solutions with the temperature is in accordance with results obtained in previous investigations using spectrophotometric ${ }^{2}$ and acidimetric ${ }^{10}$ methods.

Equilibrium constants for the various polysulfide ions. The results obtained in a previous spectrophotometric study of aqueous polysulfide solutions could be satisfactorily described with some, slightly different, models for the equilibria between polysulfide and hydrogen sulfide ions. ${ }^{2}$ The equilibrium constants obtained were given in terms of the $\mathrm{S}_{4} \mathrm{~S}^{2-}$ ion as

$$
K_{i / 4}=\left[\mathrm{S}_{i} \mathrm{~S}^{2-}\right]\left[\mathrm{S}_{4} \mathrm{~S}^{2-}\right]^{-i / 4}\left(\left[\mathrm{HS}^{-}\right]\left[\mathrm{OH}^{-}\right]\right)^{1-i / 4}
$$

As none of the solutions previously studied were in equilibrium with elementary sulfur, the equilibrium constants could not be expressed in terms of elementary sulfur, i.e. in the form of eqn. (4). 
By combining the information obtained in the present and in the previous spectrophotometric investigation, ${ }^{2}$ the relative equilibrium constants of type (6) of the various equilibrium models could be transformed, by calculating the concentrations of the ions for the conditions on the sulfur-polysulfide equilibrium curve, into "individual" constants of type (4) for the various polysulfide ions. The calculations were carried out on a digital computer, Control Data CD 3600, using the HALTAFALL programme. ${ }^{11}$ The equilibrium constants, $K_{i}$, obtained for the different models, and the $\bar{n}$ values at equilibrium with elementary sulfur, $\bar{n}_{\mathrm{e}}$, are given in Tables 1 and 2 . The standard deviation

Table 1. Equilibrium models for polysulfide solutions at $25^{\circ} \mathrm{C}$. The logarithms of the equilibrium constants $K_{i}$, eqn. (4), of the various polysulfide ions, together with the $\bar{n}$ and $B$ values for polysulfide solutions in equilibrium with elementary sulfur, denoted $\bar{n}_{\mathrm{e}}$ and $B_{\mathrm{e}}$, are given. Different equilibrium hypotheses are denoted by the $n$ 's of the species $\mathbf{S}_{n} \mathbf{S}^{2-}$ considered present.

\begin{tabular}{|c|c|c|c|}
\hline Hypothesis & $3,4,5$ & $2,3,4,5$ & $2,3,4,5,6$ \\
\hline $\begin{array}{l}\log K_{2} \\
\log K_{3} \\
\log K_{4} \\
\log K_{\mathrm{s}} \\
\log K_{6} \\
n_{\mathrm{e}} \\
B_{\mathrm{e}}(\mathrm{M})\end{array}$ & $\begin{array}{c}\overline{3.82} \\
4.69 \\
4.47 \\
\overline{-} \\
4.27 \\
1.0 \times 10^{-6}\end{array}$ & $\begin{array}{c}2.25 \\
3.93 \\
4.59 \\
4.57 \\
\overline{-34} \\
4.3 \times 10^{-6}\end{array}$ & $\begin{array}{c}2.14 \\
3.96 \\
4.48 \\
4.60 \\
2.32 \\
4.39 \\
1.5 \times 10^{-6}\end{array}$ \\
\hline
\end{tabular}

Table 2. Equilibrium models for polysulfide solutions at $80^{\circ} \mathrm{C}$. The logarithms of the equilibrium constants $K_{i}$, eqn. (4), of the various polysulfide ions, together with the $n$ and $B$ values for polysulfide solutions in equilibrium with elementary sulfur, denoted $\bar{n}_{\mathrm{e}}$ and $B_{\mathrm{e}}$, are given. Different equilibrium hypotheses are denoted by the $n$ 's of the species $\mathrm{S}_{n} \mathrm{~S}^{2-}$ considered present.

\begin{tabular}{|c|c|c|c|}
\hline Hypothesis & $3,4,5$ & $2,3,4,5$ & $2,4,5,6$ \\
\hline $\begin{array}{c}\log K_{\mathbf{2}} \\
\log K_{3} \\
\log K_{4} \\
\log K_{5} \\
\log K_{\mathrm{b}} \\
\\
n_{\mathrm{e}} \\
B_{\mathrm{e}}(\mathrm{M})\end{array}$ & $\begin{array}{c}3 . \overline{24} \\
4.45 \\
4.26 \\
\overline{-} \\
4.35 \\
2.1 \times 10^{-6}\end{array}$ & $\begin{array}{c}1.87 \\
3.49 \\
4.42 \\
4.30 \\
- \\
4.34 \\
2.0 \times 10^{-6}\end{array}$ & $\begin{array}{c}2.04 \\
- \\
3.49 \\
4.33 \\
3.47 \\
5.02 \\
2.8 \times 10^{-6} \\
\end{array}$ \\
\hline
\end{tabular}

for the $\log K_{i}$ and $\bar{n}_{\mathrm{e}}$ values, calculated from various experimental points on the equilibrium curve, is about 0.01 . The only exception is the model $2,4,5,6$ at $80^{\circ} \mathrm{C}$ (Table 2), where the standard deviation for $\log K_{i}$ was about 0.1 ; the standard deviation for $\bar{n}$ was, however, still 0.01 . In this model, the species $\mathrm{S}_{3} \mathrm{~S}^{2-}$ was rejected by the computer in calculating the equilibrium constants. ${ }^{2}$

Acta Chem. Scand. 25 (1971) No. 5 
This might be erroneous. The previous equilibrium analysis ${ }^{2}$ indicated minor systematic experimental errors in the spectrophotometric measurements at the higher temperature.

The equilibrium constants of all the polysulfide ions decreased with increasing temperature, irrespective of the model used. The $B_{\mathrm{c}}$ and $\bar{n}_{\mathrm{e}}$ values varied directly with the temperature, in accordance with the results obtained by plotting $1 / X_{\mathrm{s}}$ against $1 /\left[\mathrm{OH}^{-}\right]$. The $B_{\mathrm{e}}$ and $\bar{n}_{\mathrm{e}}$ values obtained from the equilibrium models were, however, somewhat lower than the ones obtained by plotting, especially at $80^{\circ} \mathrm{C}$. This is probably the result of neglecting the presence of very large polysulfide ions in the equilibrium models. The presence of these is also indicated by the comparatively bad description by the equilibrium models, especially when $\mathrm{S}_{6} \mathrm{~S}^{2-}$ was also excluded, of the high alkalinityhigh $X_{s}$ part of the aqueous polysulfide-elementary sulfur equilibrium curve. It should be remembered that the equilibrium constants were determined from spectrophotometric measurements on polysulfide solutions not in equilibrium with elementary sulfur, i.e. at larger $B$ and smaller $\bar{n}$, conditions at which the concentrations of the very large polysulfide ions are negligible.

Acknowledgements. The author is indebted to Professor Nils Hartler, head of the Pulp Technology Department of STFI, for his interest in this work, to Mrs. Lilian Gustafsson and Miss Disa Tormund for skilful experimental work, and to the Royal Institute of Technology, Stockholm, for computer time.

\section{REFERENCES}

1. Arntson, R. H., Dickson, F. W. and Tunell, G. Science 128 (1958) 716.

2. Teder, A. Arkiv Kemi 31 (1969) 173.

3. Teder, A. Svensk Papperstid. 68 (1965) 825.

4. Teder, A. Svensk Papperstid. 71 (1968) 149.

5. Teder, A. Svensle Papperstid. 72 (1969) 294.

6. Ahlgren, P. Svensk Papperstid. 70 (1967) 730.

7. Teder, A. Svensk Papperstid. 70 (1967) 197.

8. Teder, A. Svensk Papperstid. 72 (1969) 245.

9. Gustafsson, L. and Teder, A. Svensk Papperstid. 72 (1969) 249.

10. Gustafsson, L. and Teder, A. Svensk Papperstid. 72 (1969) 795.

11. Ingri, N., Kakolowicz, W., Sillén, L. G. and Warnqvist, B. Talanta 14 (1967) 1261.

Received September 30, 1970. 